\title{
Link-Level Performance of Indoor Body Area Networks with Centralized Topologies
}

\author{
J.-M. Dricot ${ }^{1}{ }^{*}$, S. Van Roy $^{1}$, G. Ferrari ${ }^{2}$, Fr. Horlin ${ }^{1}$ and Ph. De Doncker ${ }^{1}$ \\ ${ }^{1}$ Université Libre de Bruxelles OPERA -- Wireless Communications Group, Belgium \\ ${ }^{2}$ University of Parma, Italy WASN Lab, Department. of Information Engineering, Italy
}

\begin{abstract}
BANs) are key building blocks of the future generation networks and the Internet of Things as well. In the last years, research has focused on channel modeling and on the design of efficient medium access control (MAC) mechanisms. Less attention has been paid to network-level performance analysis. Thereby, this paper presents a novel analytical framework for network performance analysis with star (i.e., centralized) topologies. This framework takes into account realistic channel statistics and provides several insights on BAN design and analysis.
\end{abstract}

Keywords: Body area network (BAN), medium access control (MAC) protocol, link level performance analysis.

\section{INTRODUCTION}

Recent advances in ultra-low powered sensors have fostered the research in the field of body-centric networks, also referred to as body area networks (BANs). In these networks, a set of nodes (called sensors) are deployed on the human body. Their objective is to monitor and report several physiological values: blood pressure, breathing rate, skin temperature, or heart rate.

Generally, sensing is performed at low rates but in emergencies, the network load may increase within seconds. Therefore, an in-depth analysis of the network outage, throughput, and achievable transmission rate can provide insight on the maximum supported reporting rate and the corresponding performance.

The focus of this paper is on link-level performance of BANs and the integration of the propagation channel characteristic in a general network-level performance analysis framework. All considered networks would have star topologies, meaning the sensor nodes will be directly connected to a central controller. The modeling of the BAN channel was recently thoroughly investigated [1-5]. The main findings on the body-radio propagation channel can be summarized. First, the average value of power decreases as an exponential function of the distance; however, unlike classical propagation models, where the received power $P$ is a decreasing function of the form distance $d^{-\alpha}$, in [6], the authors show that a law of the form $10^{\gamma d}(\gamma<0)$ more accurately characterizes body-radio propagation. Second,

*Address correspondence to this author at the Department OPERA -- Wireless Communications Group, Université Libre de Bruxelles; Belgium

Tel: +32-2-6503883; Fax: +32-2-6504713; E-mail: jdricot@ulb.ac.be the propagation channel is subject to distinct propagation mechanisms with respect to the location of the sensors on the body. More precisely, on-body propagation and reflections from the environment combine to create a particular propagation mechanism that is specific to BANs.

This paper addresses the development of a specific framework for accurately evaluating the node throughput for BANs, with this metric being a traditional measure of how much traffic can be delivered, per time unit, by the network $[7,8]$. Therefore, our analysis is practical for understanding the level of information that could be collected and processed in body-related applications (e.g., health or fitness monitoring).

The slotted and asynchronous communications are designed so that in every time slot, each node independently transmits with a probability of $q$. Indeed, in a generic scenario, the traffic distribution in a sensor network can be considered temporally bursty. In other words, body areas may vary temporally, either with high traffic loads, or with other areas with little or no traffic, or even with a scheduled sleep for the nodes. Therefore, in a first approximation, a random medium access protocol, such as the ALOHA scheme presented here, could be used to keep the amount of coordination traffic low and provide a straightforward implementation suitable for computation-constrained sensor nodes.

The remainder of this paper is organized as follows. In Section 2, the models, definitions, and notations related to propagation mechanisms are introduced. In Section 3, the conditional success probability of a transmission for a node, given the transmitter-receiver and interference-receiver distances, is derived. Section 4 investigates the minimum 
Table 1. Main Mathematical Symbols and Variables in Use

\begin{tabular}{|c|c|c|}
\hline Symbol & Description & Units \\
\hline$X$ & random variable $\mathbf{X}$ & \\
\hline$E[X]$ & expectation of the random variable $\mathbf{X}$ & \\
\hline$P\{E\}$ & probability of event $\mathrm{E}$ & \\
\hline$d$ & transmission distance & $\mathrm{m}$ \\
\hline$P$ & average transmit power & $\mathrm{W}$ \\
\hline $\mathbf{P}$ & instantaneous transmit power & $\mathrm{W}$ \\
\hline$P(d)$ & average received power at distance $d$ & $\mathrm{~W}$ \\
\hline $\mathbf{P}(d)$ & instantaneous received power at distance $d$ & $\mathrm{~W}$ \\
\hline$P_{\text {int }}$ & instantaneous total interference power & $\mathrm{W}$ \\
\hline$P_{\text {env }}$ & instantaneous interf. power from the environment & $\mathrm{W}$ \\
\hline$P_{\text {env }}$ & average interf. power from the environment & $\mathrm{W}$ \\
\hline$L(d)$ & power loss at distance $d$ & $\mathrm{~dB}$ \\
\hline$L_{\text {ref }}$ & power loss at reference distance & $\mathrm{dB}$ \\
\hline$d_{\text {ref }}$ & reference distance & $\mathrm{m}$ \\
\hline$\gamma$ & path loss constant & $\mathrm{dB} / \mathrm{m}$ \\
\hline SINR & instantaneous signal-to-interference-and-noise ratio & $\mathrm{dB}$ \\
\hline$\theta$ & threshold SINR of a communication link & $\mathrm{dB}$ \\
\hline$P_{\text {th }}$ & threshold link probability of success & \\
\hline$\lambda$ & instantaneous transmission state of a node & \\
\hline$q$ & node probability of transmission & \\
\hline$\tau$ & probabilistic link throughput & \\
\hline$P_{0}, d_{0}$ & subscript " 0 " refers to the link of interest & \\
\hline$P_{i}, P_{j}$ & subsrcipts $\boldsymbol{i}$ or $\boldsymbol{j}$ refer to the interferers & \\
\hline$d_{i}, d_{j}$ & & \\
\hline
\end{tabular}

required transmission power and the average link throughput for centralized topology. Section 5 concludes the paper.

\section{PROPAGATION MECHANISMS OF BANS AND STOCHASTIC CHANNEL MODELING}

Recent research results in the field of human body-radio propagation have highlighted the following three distinct propagation mechanisms taking place in the context of onbody communications [9].

First, there is propagation through the body; however, when high transmission frequencies are considered, the attenuation undergone by these waves is relevant and the corresponding contribution can be neglected.

A second mechanism corresponds to guided diffraction around the body. This mechanism is consistent with surface wave propagation and its properties depend on the body specific characteristics.
Finally, the last propagation contribution comes from the surrounding environment. More precisely, this propagation mechanism originates from reflections off the body's limbs (arms and legs) and the surrounding objects (walls, floor, and ceiling).

The experimental setup used in this manuscript is presented in Fig. (1) and the main mathematical symbols and variables in use in the expressions are introduced in Table $\mathbf{1}$. We now present accurate statistical models corresponding to the propagation mechanisms previously described

\subsection{On-Body Propagation (Guided Diffraction)}

As previously emphasized in $[6,10]$, the average received power (in $\mathrm{dB}$ scale) is the following linearly decreasing function of the distance:

$\mathrm{E}[\mathrm{P}(d)]=P+L_{\mathrm{ref}}+10 \gamma\left(d-d_{\mathrm{ref}}\right) \quad d \geq d_{\mathrm{ref}}$ 

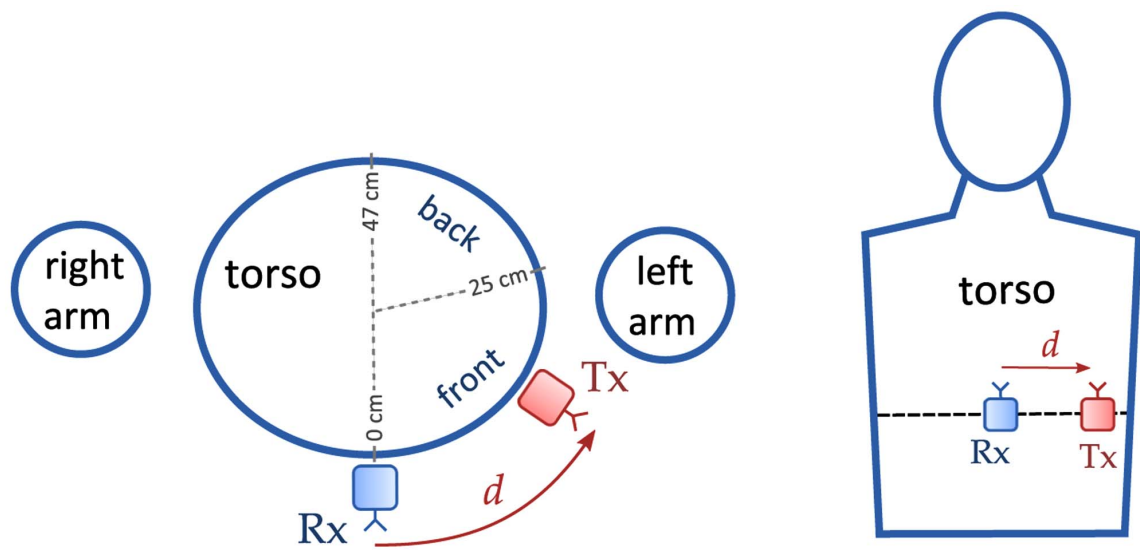

Fig. (1). Possible positions of a transmitter-receiver pair in a BAN.

where $P(d)$ is the instantaneous received power (units: [W]) at distance $d$ (units: [m]), $P$ the initial transmission power (units: $[\mathrm{W}]$ ), $d_{\text {ref }}$ is a reference distance (units: [m]), $L_{\text {ref }}$ is the loss at the reference distance (adimensional, in $\mathrm{dB}$ ), and $\gamma$ is a suitable constant ( units: $[\mathrm{dB} / \mathrm{m}]$ ). For instance, typical experimental values for these parameters are $d_{\text {ref }}=8 \mathrm{~cm}, L_{\text {ref }}=-57.42 \mathrm{~dB}$, and $\gamma=-124 \mathrm{~dB} / \mathrm{m}$ [10].

The average received power, in linear scale, can then be expressed as follows:

$\mathrm{E}[\mathrm{P}(d)]=P \cdot L(d) \quad d \geq d_{\mathrm{ref}}$

where

$$
\begin{aligned}
& \mathrm{L}(\mathrm{d})=\underbrace{10^{\left(\mathrm{L}_{\text {ref }}-10 \gamma \mathrm{d}_{\text {ref }}\right) / 10}}_{=\mathrm{L}_{0}} \cdot 10^{\gamma \mathrm{d}}=\mathrm{L}_{0} 10^{\gamma \mathrm{d}} \quad \mathrm{d} \geq \mathrm{d}_{\text {ref }} \\
& =L_{0} 10^{\gamma d} \quad d \geq \mathrm{d}_{\mathrm{ref}}
\end{aligned}
$$

where $L_{0}$ is a function of $L_{\text {ref }}, d_{\text {ref }}$, and $\gamma .{ }^{1}$ In Fig. (2) (a), the loss $L$ is shown as a function of the distance, considering narrowband transmissions at $5 \mathrm{GHz}$.

More precisely, in Fig. (2) (a) experimental measurements (circles) and their linear interpolation (solid line) are shown. Finally, using (3) in (2) one obtains:

$$
\mathrm{E}[\mathrm{P}(d)]=P \cdot L_{0} 10^{\gamma d}
$$

While expression (4) characterizes the average value, it does not provide insights on the instantaneous distribution of the received power. In [10], it has been experimentally observed that the on-body propagation channel is characterized by slow large-scale fading (i.e., shadowing). More precisely, the instantaneous received power at distance $d$ can be expressed as follows:

$\mathbf{P}(d)=P L_{0} 10^{\gamma d} \mathbf{X}$

where $\mathbf{X}$ is a random variable (RV) which depends on the channel characteristics. It is shown in [11] and confirmed by

${ }^{1}$ Note that, since (3) holds for $d \geq d_{\text {ref }}, L_{0}$ can be intuitively interpreted as the (extrapolated) loss (adimensional, linear scale) at distance $d=d_{\text {ref }}$. In other words, $L_{0}$ takes into account the loss due to antenna emission. our measurements that $\mathbf{X}$ has a log-normal distribution ${ }^{2}$ with parameters $\mu$ and $\sigma$, where $\sigma_{\mathrm{dB}}$ typically ranges from
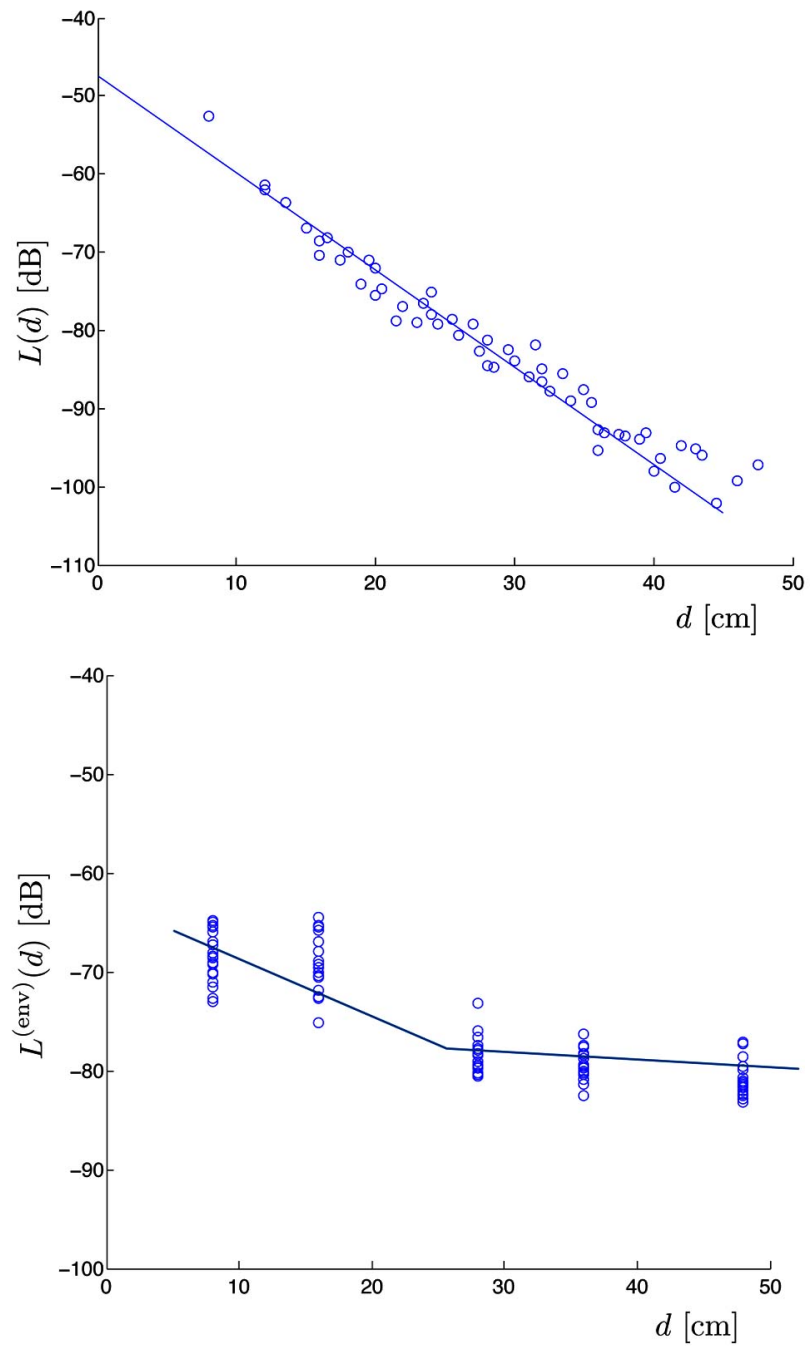

Fig. (2). On-body propagation loss as a function of the distance: experimental results (circles) and linear interpolation (solid line).

$4 \mathrm{~dB}$ to $10 \mathrm{~dB}, \mu_{\mathrm{dB}}$ is the average path loss on the link

${ }^{2}$ Note that we use the $\log _{10}$ variant of the log-normal since the widely-used shadowing model uses an additive Gaussian variation expressed in $\mathrm{dB}$. 


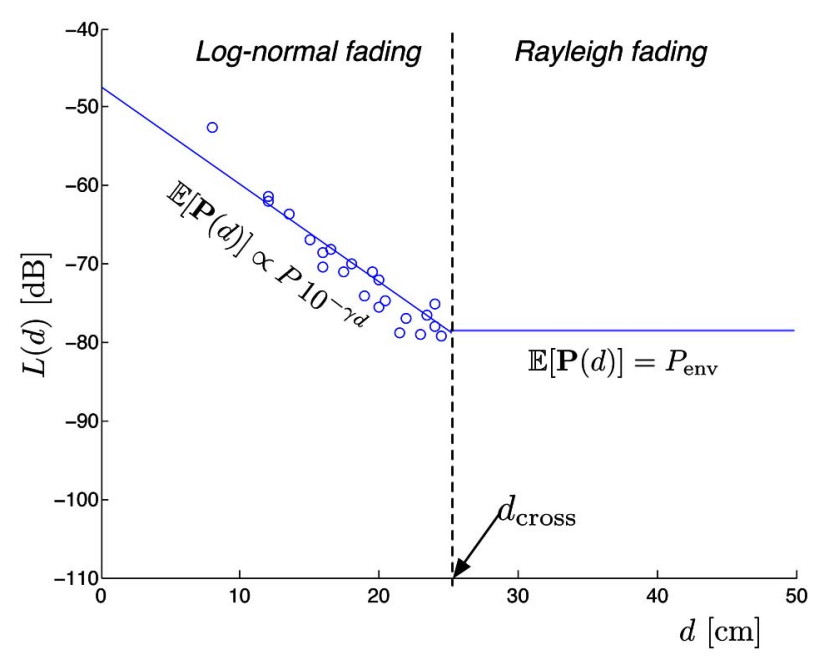

Fig. (3). Generic propagation model (on-body and environment reflection superimposed)

(units: $[\mathrm{dB}]$ ). Since the loss is accounted for by the term $L(d)$, it follows that $\mu_{\mathrm{dB}}=0$ and the cumulative distribution function (cdf) of $X$, i.e., $P\{X \leq x\}$, reduces to the following: ${ }^{3}$

$$
F_{\mathrm{X}}(X ; 0, \sigma)=\frac{1}{2}-\frac{1}{2} \operatorname{erf}\left(\frac{-10 \log _{10} x}{\sigma \sqrt{2}}\right)
$$

with the following corresponding probability density function (pdf):

$$
f_{\mathrm{x}}(x ; 0, \sigma)=\frac{10}{(\ln 10) x \sqrt{2 \pi} \sigma} \exp \left\{-\frac{\left(10 \log _{10} x\right)^{2}}{2 \sigma^{2}}\right\}
$$

\subsection{Reflections from the Environment}

The second significant propagation mechanism originates form the multiple reflections from the environment, i.e., the reflections and the diffractions taking place on the floor, the ceiling, the walls, and the furnitures, among others. A substantial measurement campaign conducted by the authors (and detailed in $[6,9,10]$ ) has shown that the contribution of the environment can be considered, on average, as an additive, constant power when the transmission distance is significant (i.e., when $d>25 \mathrm{~cm}$ ). The obtained results are shown in Fig. (2) (b), the power received by means of reflections from the surrounding environment is shown as a function of the distance. It can be observed that when $d>25 \mathrm{~cm}$, the value of the loss is, on average, around -78 $\mathrm{dB}$. More precisely, for $d>25 \mathrm{~cm}$ the average value of the received power can be expressed, in logarithmic scale, as follows:

$$
E\left[\mathrm{P}_{\mathrm{cuv}}\right]-P_{\mathrm{eav}}=P+L_{\mathrm{db}}^{(\mathrm{eav})}
$$

where $P$ is the transmit power and $L_{\mathrm{dB}}^{(\mathrm{env})}=-78 \mathrm{~dB}$. Alternatively, the average received power can be expressed in linear scale as

\footnotetext{
${ }^{3}$ In (6) and (7), for the sake of clarity the mean and standard deviation are explicitly indicated at the right-hand sides.
}

$E\left[\mathrm{P}_{\mathrm{cav}}\right]-P_{\mathrm{cav}}=P \cdot L^{(\mathrm{cav})}$

where

$$
L^{\text {(env) }}=10^{L_{\mathrm{dB}}^{(\text {env) }} / 10} .
$$

Our measurement campaign has shown that the propagation channel can be accurately characterized as narrowband Rayleigh block fading. Therefore, the instantaneous received power $\mathbf{P}$ env has the following exponential distribution [12]:

$$
f_{P_{\text {env }}}(x)=\frac{1}{P_{\text {env }}} \exp \left\{-\frac{x}{P_{\text {env }}}\right\} \text {. }
$$

\subsection{A Unified BAN Propagation Model}

The combination of the two effects presented in Subsection 2.1 and Subsection 2.2 allows to derive a unified propagation model for a generic BAN. It can be observed that the degree of importance of each mechanism depends on the distance between transmitter and receiver. More precisely, in close proximity, the dominant propagation mechanism is the on-body propagation described in Subsection 2.1. Above the cross-over distance $d_{\text {cross }} \approx 25$ $\mathrm{cm}$, the contribution of the environment becomes dominant and the second propagation mechanism, presented in Subsection 2.2, is more accurate.

Therefore, a unified propagation model can be characterized as follows:

- if $d \leq d_{\text {cross }}$, the average received power can be computed using (4) (i.e., $\mathrm{E}[\mathrm{P}(d)] \propto P 10^{\gamma d}$ ) and the instantaneous received power is determined by the lognormal fading channel model given by (7);

- if $d>d_{\text {cross }}$, the average received power approximately is constant (i.e., $\mathrm{E}[\mathrm{P}(d)]=P L^{\text {(env) }}$ ) and the instantaneous received power, owing to a Rayleigh faded channel model, has the distribution given by (10).

In Fig. (3), the average path loss is shown as a function of the distance.

In particular, the overall (unified) path loss can be expressed as follows:

$$
L(d)=\max \left\{L_{0} 10^{\gamma d}, L^{(\text {env) }}\right\} \text {. }
$$

\section{LINK-LEVEL NETWORK PERFORMANCE IN A MULTI-USER SCENARIO}

In this paper, we consider multi-user communications--in a BAN, all sensors need to transmit to a central controller and, in this sense, the scenario at hand can be interpreted as a multi-user scenario. The transmission of interest is denoted with the subscript " 0 ." Depending on their distance to the receiver, the interfering sensor nodes will be denoted differently. More precisely:

- the interferers located at distances shorter than $d_{\text {cross }}$ are referred to as "close-range interferers," their number is indicated as $N$, and they are denoted with the subscript $i \in \mathrm{N}=\{1,2, \quad, N\}$; 
- the interferers located at distances longer than $d_{\text {cross }}$ are referred to as "far-range interferers," their number is indicated as $N^{\prime}$, and they are denoted with the subscript $j \in \mathrm{N}^{\prime}=\left\{1,2, \quad, N^{\prime}\right\}$.

The transmission state of the a node at time $t$ is characterized by the following indicator variable:

$\Lambda(t)= \begin{cases}1 & \text { if the node istransmitting at time } t \\ 0 & \text { if the node is silent at time } t\end{cases}$

Assuming slotted transmissions (i.e., $t$ can assume multiples of the slot time), a simple random access scheme is such that, at each time slot, a node transmits with probability $q$

[13, p.278]. Therefore, $\quad\left\{\lambda_{i}(t)\right\}_{t=1}^{\infty}, i \in \mathrm{N} \quad$ and

$\left\{\lambda_{j}(t)\right\}_{t=1}^{\infty}, j \in \mathrm{N}^{\prime}$ are sequences of Bernoulli RVs with $\mathrm{P}\left\{\lambda_{i}(t)=1\right\}=\mathrm{P}\left\{\lambda_{j}(t)=1\right\}=q, \forall t i, j$.

A transmission in a given link is successful if and only if the signal-to-noise and interference ratio (SINR) at the receiver is above a certain threshold $\theta$. This threshold value depends on the receiver characteristics, the modulation format, and the coding scheme, among other aspects. The SINR at the receiving node of the link is given by

$\operatorname{SINR}-\frac{P_{i l}\left(d_{1}\right)}{N_{0} B+P_{\text {int }}}$

where $P_{0}\left(d_{0}\right)$ is the received power from the link source located at distance $d_{0}, N_{0}$ is the power noise spectral density, $B$ the channel bandwidth, and $\mathrm{P}_{\text {int }}$ is the total interference power at the link receiver, i.e., the sum of the instantaneous received powers from all the undesired transmitters:

$$
P_{\mathrm{int}}=\sum_{i=1}^{N} \Lambda \cdot P \cdot\left(d_{i}\right)+\sum_{j=1}^{N} \Lambda_{j} P_{c: 1.1}
$$

where the variables $\mathbf{P}_{i}$ and $d_{i}$ refer to the power and the distance-to-receiver of the $i$-th node, respectively. Finally, as typical in the context of BANs, we assume that all nodes use the same transmit power, i.e., $P_{i}(0)=P_{j}(0)=P_{0}(0), \forall i, j$.

We now provide the reader with the derivations of the link probability of success in the two propagation mechanisms observed in BANs and detailed in Subsection 2.1 and Subsection 2.2. We underline that the analytical framework deriveed in the following subsections is expedient to grasp a preliminary understanding of the behaviour of a BAN. As the analytical framework relies on realistic assumptions, based on an extensive measurement campaign, the obtained results are meaningful. Experimental validation of the same framework with a practical BAN testbed is a relevant research activity and we are currently working on it.

\subsection{Link Probability of Success with Short-range Transmission}

The link probability of success for a required threshold SINR value $\theta$ in the context of a log-normal faded link is equal to

$$
\begin{aligned}
& \mathrm{P}\{\mathrm{SINR}>\theta\}=\mathrm{E}_{P_{\mathrm{int}}}\left[\mathrm{P}\left\{\mathrm{SINR}>\theta \quad P_{\mathrm{int}}\right\}\right] \\
& =\mathrm{E}_{P_{\mathrm{int}}}\left[\mathrm{P}\left\{\frac{P_{0} L\left(d_{0}\right) \mathrm{X}_{0}}{N_{0} B+P_{\mathrm{int}}}>\theta \quad P_{\mathrm{int}}\right\}\right] \\
& =\mathrm{E}_{P_{\text {int }}}\left[1-\mathrm{P}\left\{\mathrm{X}_{0} \leq \theta \frac{N_{0} B+P_{\mathrm{int}}}{P_{0} L\left(d_{0}\right)} P_{\mathrm{int}}\right\}\right] \\
& =\mathrm{E}_{P_{\text {int }}}\left[\frac{1}{2}+\frac{1}{2} \operatorname{erf}\left(\frac{-10}{\sigma \sqrt{2}} \log _{10}\left(\theta \frac{N_{0} B+P_{\text {int }}}{P_{0} L\left(d_{0}\right)}\right)\right)\right] .
\end{aligned}
$$

where, in the last passage, we exploited the fact that the cumulative distribution function of the random variable $X_{0}$ is given by (6). In the Appendix, it is shown that

$\zeta(Z \sigma)=\frac{1}{2}+\frac{1}{2} \operatorname{er}\left(\frac{-10 \log _{10} z}{\sigma \sqrt{2}}\right) \approx \sum_{k}^{n} c_{k} \exp \left(-a_{k} z\right)$

where $\left\{c_{k}\right\}_{k=1}^{n}$ and $\left\{-a_{k}\right\}_{k=1}^{n}$, where $n$ is an integer determined by the expansion accuracy, are suitable coefficients. By using the function $\zeta(\cdot ; \cdot)$ and recalling expressions (13) and (5) for the interference power, the link probability of success (14) can be written as follows:

$$
\begin{aligned}
& \mathrm{P}\{\mathrm{SINR}>\theta\}=\mathrm{E}\left[\sum_{k=1}^{n} c_{k} \exp \left(\frac{-a_{k} \theta N_{0} B}{P_{0} L\left(d_{0}\right)}\right)\right. \\
& \left.\times \exp \left(-a_{k} \theta \sum_{i=1}^{N} \frac{L\left(d_{i}\right)}{L\left(d_{0}\right)} \mathrm{X}_{i} \lambda_{i}\right) \times \exp \left(-a_{k} \theta \sum_{j=1}^{N} \frac{P_{\text {env }}}{P_{0} L\left(d_{0}\right)} \lambda_{j}\right)\right] \\
& =\sum_{k=1}^{n} c_{k} \exp \left(\frac{-a_{k} \theta N_{0} B}{P_{0} L\left(d_{0}\right)}\right) \times \operatorname{Eexp}\left(-a_{k} \theta \sum_{i=1}^{N} \frac{L\left(d_{i}\right)}{L\left(d_{0}\right)} \mathrm{X}_{i} \lambda_{i}\right) \\
& \times \operatorname{Eexp}\left(-a_{k} \theta \sum_{j=1}^{N} \frac{P_{\text {env }}}{P_{0} L\left(d_{0}\right)} \lambda_{j}\right)
\end{aligned}
$$

where, in the last passage, we have used the fact that the RVs $\left\{\Lambda_{i}, \Lambda_{j}, P_{\text {env }}, X_{i}\right\}$ are independent and the term $C_{k} \exp (\cdot)$ is constant. The term in the second line of the expression at the right-hand side of (16) can be further expressed as

$$
\begin{aligned}
& \mathrm{E} \exp \left(-a_{k} \theta \sum_{i=1}^{N} \frac{L\left(d_{i}\right)}{L\left(d_{0}\right)} \mathbf{X}_{i} \lambda_{i}\right) \\
& =\mathrm{E} \prod_{i=1}^{N} \exp \left(-a_{k} \theta \frac{L\left(d_{i}\right)}{L\left(d_{0}\right)} \mathbf{X}_{i} \lambda_{i}\right) \\
& =\prod_{i=1}^{N} \mathrm{E} \exp \left(-a_{k} \theta \frac{L\left(d_{i}\right)}{L\left(d_{0}\right)} \mathbf{X}_{i} \lambda_{i}\right)
\end{aligned}
$$

since the RVs are independent. This total expectation can be expressed by recalling the definition of the continuous random variables $X_{i}$ (given in (6)) and the discrete indicator variable $\lambda_{i}$ (given in Section 3). The relation (17) becomes:

$$
=\prod_{i=1}^{N}\left[P\left\{\lambda_{i}=0\right\} \times E \exp (0)\right.
$$




$$
\begin{aligned}
& \left.+\mathrm{P}\left\{\lambda_{i}=1\right\} \times E \exp \left(-a_{k} \theta \frac{L\left(d_{i}\right)}{L\left(d_{0}\right)} \mathbf{X}_{i}\right)\right] \\
& =\prod_{i=1}^{N}(1-q)+q \int_{0}^{\infty} \exp \left(-a_{k} \theta 10^{\gamma\left(d_{i}-\phi_{0}\right)} x\right) f_{\mathrm{x}}(x) \mathrm{d} x .
\end{aligned}
$$

Note that, in the last passage, the integral arises from the definition of the expectation of the random variable $X_{i}$. Furthermore, since all RVs $X_{i}$ are non-correlated we can note $f_{x i}(x)=f_{x}(x)$, which is defined in (7). The final integral expression in (18) can be numerically computed. The term in the third line of expression can be expressed by following the same approach used to compute (17). It yields:

$$
\begin{aligned}
& \mathrm{E} \exp \left(-a_{k} \theta \sum_{j=1}^{N^{\prime}} \frac{\mathrm{P}_{\mathrm{env}}}{P_{0} L\left(d_{0}\right)} \lambda_{j}\right) \\
& =\prod_{j=1}^{N^{\prime}} \mathrm{E} \exp \left(-a_{k} \theta \frac{\mathrm{P}_{\mathrm{env}}}{P_{0} L\left(d_{0}\right)} \lambda_{j}\right) \\
& =\prod_{j=1}^{N^{\prime}}\left[\mathrm{P}\left\{\lambda_{j}=0\right\} \times \mathrm{E} \exp (0)\right. \\
& \left.+\mathrm{P}\left\{\lambda_{j}=1\right\} \times \mathrm{E} \exp \left(-a_{k} \theta \frac{\mathrm{P}_{\mathrm{env}}}{P_{0} L\left(d_{0}\right)}\right)\right] \\
& \left.=[1-q)+q \int_{0}^{\infty} \exp \left(-a_{k} \theta \frac{x}{P_{0} L\left(d_{0}\right)}\right) \frac{1}{P_{\mathrm{env}}} e^{-x / P_{\mathrm{env}} \mathrm{d} x}\right]^{N^{\prime}} \\
& =\left[\begin{array}{c}
\left.\frac{L_{0} 10^{\gamma \theta_{0}}}{L^{(\mathrm{env})}}+\theta\right]^{N^{\prime}} \\
1-
\end{array}\right.
\end{aligned}
$$

Finally, by using (18) and (19) into (16) the link probability of success can be given by the expression in (20).

\subsection{Link Probability of Success with Long-Range Transmission}

The Rayleigh-faded channel model applies to links with length $d>d_{\text {cross }}$. In this scenario, $\mathrm{E}[\mathrm{P}(d)] \approx P_{\text {env }}$ (for both the intended transmitter and interferers) and the link probability of success can be expressed as follows:

In the second passage, we exploited the fact that, if the power of the signal is exponentially distributed, its SINR also follows an exponential distribution [12]. It can be observed that the terms in the second and third lines at the right-hand side of (22) are similar to (18) and (19). Therefore, by using the same derivation (where $P_{0} L\left(d_{0}\right)$ is suitably replaced by $P_{0} L^{(\text {env) }}$ ), one has

$$
\begin{aligned}
& \mathrm{E} \exp \left(-\theta \sum_{i=1}^{N} \frac{P_{i} L\left(d_{i}\right)}{P_{\mathrm{env}}} \mathrm{X}_{i} \lambda_{i}\right)= \\
& \prod_{i=1}^{N}\left[q \int_{0}^{\infty} \exp \left(-\theta \frac{L_{0} 10^{\gamma d_{i}}}{L^{\text {(env) }}} x\right) f_{\mathrm{x}}(x) \mathrm{d} x+(1-q)\right]
\end{aligned}
$$

and

$$
\mathrm{E} \exp \left(-\theta \sum_{j=1}^{N^{\prime}} \frac{\mathrm{P}_{\mathrm{env}}}{P_{\mathrm{env}}} \lambda_{j}\right)=\left[1-\frac{\theta q}{1+\theta}\right]^{N^{\prime}} .
$$

By inserting (23) and (24) into (22), one obtains the final expression (21) for the probability of successful transmission on the link.

\section{LINK-LEVEL PERFORMANCE OF BANS}

\subsection{Minimum Transmit Power}

The first terms in the sum at the right-hand side of (20) and at the right-hand side of (21) correspond the link probabilities of success in a noise-limited regime, i.e., when no interferers are present. In fact, setting $N=N^{\prime}=0$ (i.e., $\left.P_{\text {int }}=0\right)$ in (20) and (21), the probabilities of successful link transmission reduce to

$$
\begin{aligned}
& \left\{\mathrm{P}\{\mathrm{SINR}>\theta\}=\sum_{k=1}^{n} c_{k} \exp \left(\frac{-a_{k} \theta N_{0} B}{P_{0} L_{0} 10^{\gamma \phi}}\right)\right. \\
& =\zeta\left(\frac{\theta N_{0} B}{P_{0} L_{0} 10^{\gamma \phi}}\right) \quad \text { if } d<d_{\text {cross }} \\
& \left.P\{\operatorname{SINR}>\theta\}=\exp \left(-\frac{\theta N_{0} B}{P_{\text {env }}}\right) \text { if } d \geq d_{\text {cross }} \cdot\right\}
\end{aligned}
$$

Therefore, if a threshold link probability of success equal to $P_{\text {th }} \in(0,1)$ is required, the minimum required transmit

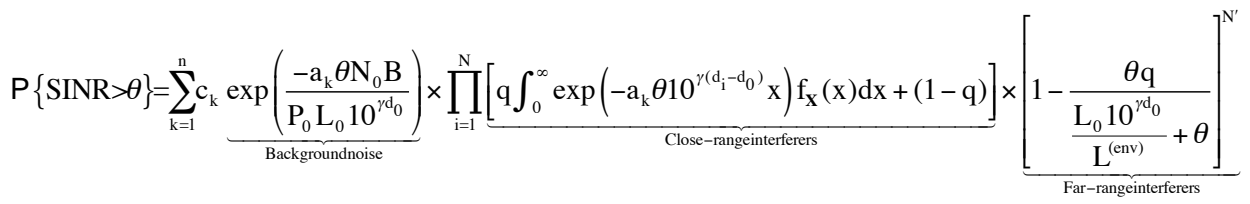

$$
\begin{aligned}
& \mathrm{P}\{\mathrm{SINR}>\theta\}=\underbrace{\exp \left(\frac{-\theta \mathrm{N}_{0} \mathrm{~B}}{\mathrm{P}_{\text {env }}}\right)}_{\text {Backgroundnoise }} \times \prod_{\mathrm{i}=1}^{\mathrm{N}}[\underbrace{\mathrm{q} \int_{0}^{\infty} \exp \left(-\theta \frac{\mathrm{L}_{0} 10^{\gamma \mathrm{d}_{\mathrm{i}}}}{\mathrm{L}^{(\text {env })}} \mathrm{x}\right) \mathrm{f}}_{\text {Close-rangeinterferers }} \mathrm{f}_{\mathbf{x}}(\mathrm{x}) \mathrm{dx}+(1-\mathrm{q})] \times \underbrace{\left[1-\frac{\theta \mathrm{q}}{1+\theta}\right]^{\mathrm{N}^{\prime}}}_{\text {Far-rangeinterferers }}
\end{aligned}
$$




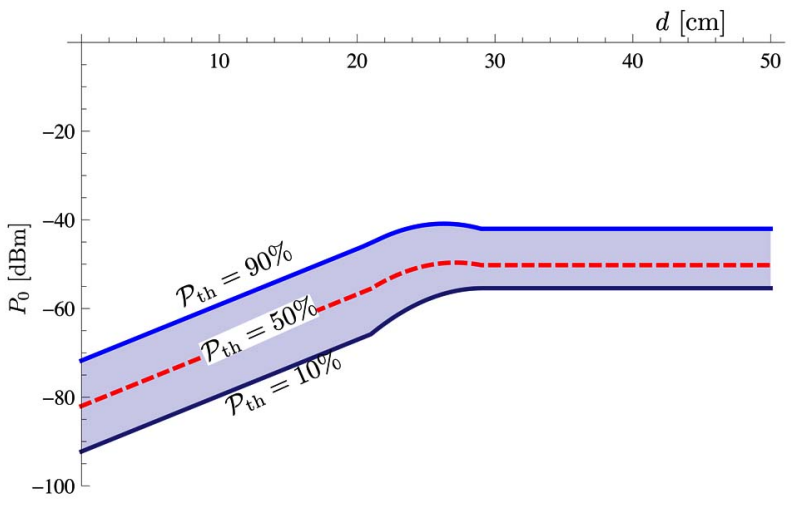

Fig. (4). Minimum transmit power as a function of the distance. The dashed region is the operational region of a BAN.

power can be written as follows:

$P_{0} \geq \begin{cases}\frac{\theta k_{\mathrm{b}} T B}{L_{0} 10^{\gamma \phi^{\gamma}} \zeta^{-1}\left(\mathrm{P}_{\mathrm{th}}\right)} & \text { if } d<d_{\text {cross }} \\ -\frac{\theta k_{\mathrm{b}} T B}{\ln \mathrm{P}_{\mathrm{th}}} & \text { if } d \geq d_{\text {coss }}\end{cases}$

where $N_{0}$ has been expressed as $T k_{\mathrm{b}}$, with $T$ being the room temperature (units: $[\mathrm{K}]$ ) and $k_{\mathrm{b}}=1.38 \times 10^{-23} \mathrm{~J} / \mathrm{K}$ being the Boltzmann's constant, and $B$ is the transmission bandwidth.

Note also that, with a slight abuse of notation, in (25) we indicate by $\zeta^{-1}(\cdot)$ the inverse of $\zeta(z \sigma)$ with respect to $\zeta$, with the implicit assumption that $\sigma$ is fixed. For instance, in Fig. (4) the minimum transmit power $P_{0}$ for a ZigBee equipment ( $B=5 \mathrm{MHz}, \theta=5 \mathrm{~dB}$ ), operating at $T=300 \mathrm{~K}$ and with log-normal fading characterized by $\sigma=4 \mathrm{~dB}$, is shown as a function of the distance, considering various values of the required link probability of success of $P_{t h}$.

It can be observed that: (i) the value of $P_{\text {th }}$ plays a limited role on the minimum transmit power; (ii) if the transmit power is constrained by energy concerns, only short-range communications (some tenths of centimeters) will be possible: a multi-hop network architecture is therefore the best choice. Finally, due to the reflections from the surrounding environment, the minimum transmit power becomes constant when $d \geq 25 \mathrm{~cm}$.

In the following subsection, we will consider only interfence-limited networks, i.e., scenarios where condition (25) is satisfied. Formally, this is equivalent to assuming that $N_{0} B=P_{\text {int }}$.

\subsection{Probabilistic Link Throughput}

A transmission is said to be successful if and only a transmission link is not in an outage, i.e., if the (instantaneous) SINR of the link is above the threshold $\theta$. The probabilistic link throughput [14] (adimensional), in the context of BANs (where transmissions can typically be organized in a full-duplex way), corresponds to the product of (i) the link probability of success and (ii) the probability $\mathrm{q}$ that the source of interest is actually transmitting, i.e.,

$$
\tau=q \times \operatorname{Prob}\{\operatorname{SINR}>\theta\}
$$

The probabilistic link throughput can be interpreted as the unconditional reception probability which can be achieved with a simple automatic-repeat-request (ARQ) scheme with error-free feedback [15].

\subsection{Performance of Analysis of a BAN with Star Topology}

In order to apply the proposed framework, we consider BANs with centralized architectures, where a central node (called $h u b$ or sink) is surrounded by and directly connected to several sensor nodes. In particular, the following two topologies are considered:

- 2 sensor nodes located at $d=10 \mathrm{~cm}$ from the sink and 2 sensor nodes at $d=30 \mathrm{~cm}$ from the sink;

- 4 sensor nodes located at $d=10 \mathrm{~cm}$ from the sink and 4 sensor nodes at $d=30 \mathrm{~cm}$ from the sink.

In Fig. (5), an illustrative representation of a BAN with

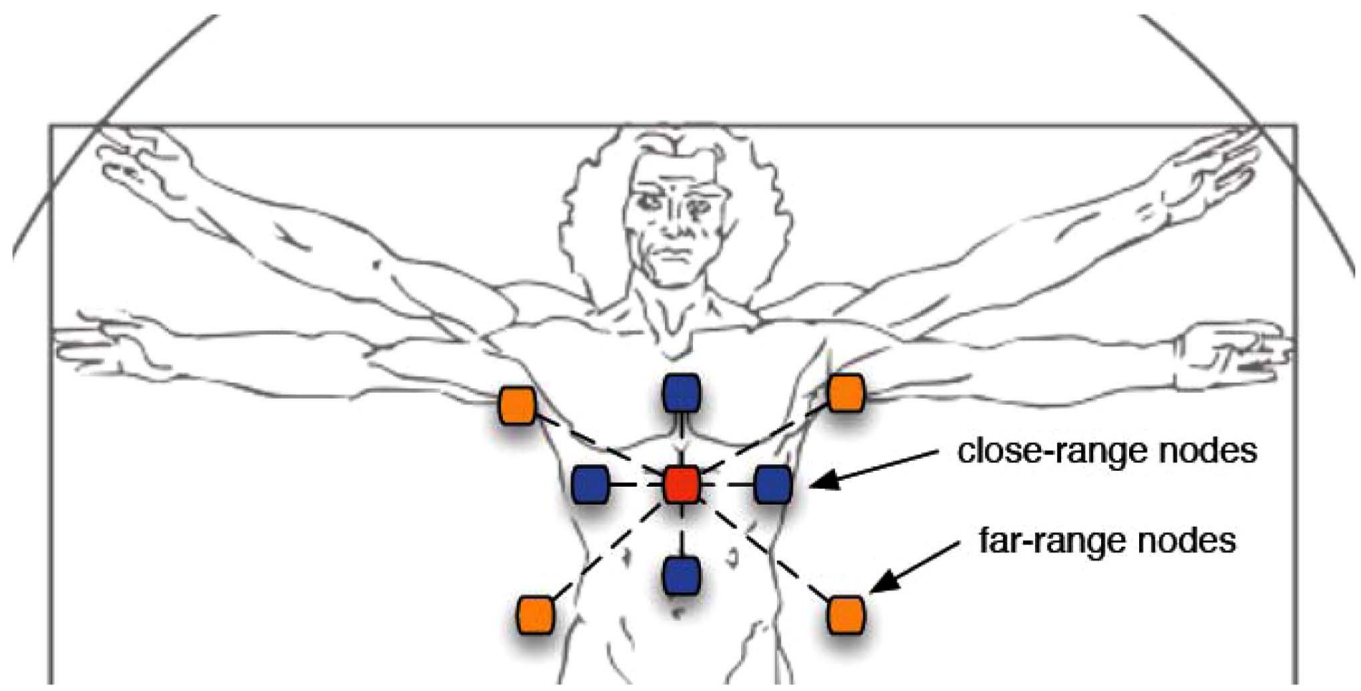

Fig. (5). Central hub (in red) surrounded by 4 close-range nodes (in blue) and 4 far-range nodes (in orange). 

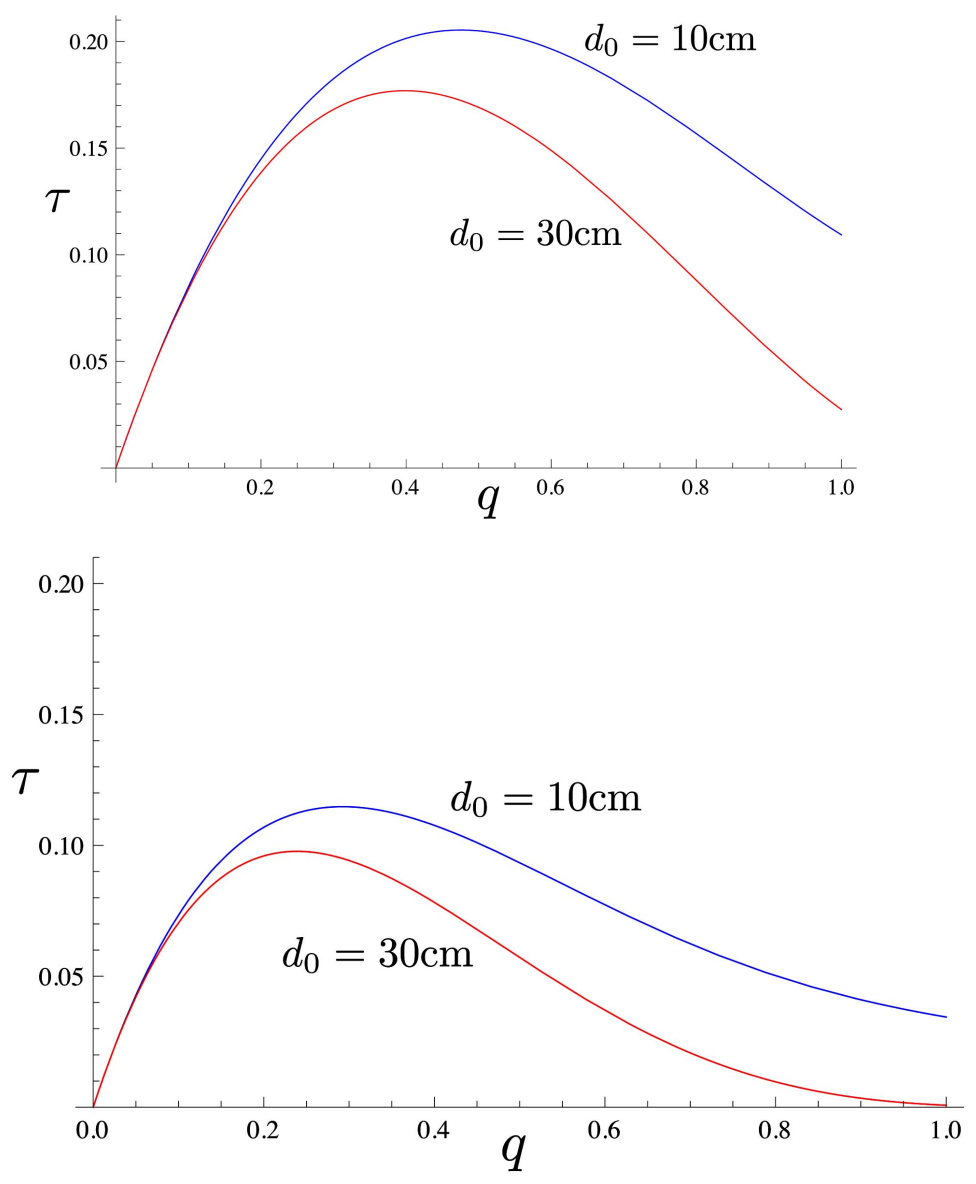

Fig. (6). Link throughput, as a function of $q$, (a) in a scenario with 2 close sensors and 2 far sensors and (b) in a scenario with 4 close sensors and 4 far sensors. In all cases, $\theta=5 \mathrm{~dB}$ and $\sigma=8 \mathrm{~dB}$.

the second topology is shown---a BAN with the first topology is simply obtained by dropping two close sensor nodes and 2 far sensor nodes from the BAN in Fig. (5).

In Fig. (6), the throughput over the link of interest is shown, as a function of the node probability of transmission (i.e., $q)$, in (a) in a scenario with $2(d=10 \mathrm{~cm})$ close sensors and 2 far $(d=30 \mathrm{~cm})$ sensors and (b) in a scenario with 4 close sensors and 4 far sensors.

In both cases, it can be observed that when the link of interest is short, i.e., the transmitter is close to the sink, the throughput is higher. This is obvious, as most of the interfering nodes are far. As expected, when the number of nodes increases, the maximum throughput achievable by a sensor node reduces. However, the relative throughput increase of a close-range node, with respect to a far-range node, remains the same (around 20\%).

Furthermore, it can be observed that, thanks to the reflections from the environment, the throughput is not significantly reduced even if the sensors are located at a long distances on the body. The difference of performance, in terms of throughput, can even be neglected at low transmission rate (i.e., when $q \leq 0.2$ ).

Finally, the proposed analytical framework allows to determine the traffic load at each sensor (in terms of probability of transmission $q$ ) which guarantees the highest achievable throughput. In particular, from the results in Fig. (6), the optimized transmission probability can be expressed as follows:

$q_{\operatorname{rax}}=\underset{a}{\operatorname{argmax} \tau}$

In the first scenario (4-node network), it can concluded that $q_{\max }=0.48$ (with corresponding throughput $\tau=0.21$ ) for the closest nodes and $q_{\max }=0.40$ (with corresponding throughput $\tau=0.18$ ) for the distant nodes. In the second scenario (8-node network), the following values are observed: $q_{\max }=0.29 \quad(\tau=0.114)$ and $q_{\max }=0.24 \quad(\tau=0.09)$ for the closest and farthest nodes, respectively.

\section{CONCLUSIONS}

In this paper, we have presented an analytical framework for the evaluation of the link probability of success in interference-limited BANs subject to fading. This analytical derivation is based on novel experimental measurements which highlight two characteristic propagation mechanisms in BANs deployed in indoor scenarios: on-body propagation (within a cross-over distance $d_{\text {cross }} ; 25 \mathrm{~cm}$ ) and propagation through reflections from the environment (limbs and surrounding objects). The obtained results show that in a BAN the very specific propagation mechanisms in presence compensate the impact of the distance. More precisely, nodes located at very different distances do not exhibit a 
Table 2. Coefficients for the Approximation of the $\zeta$ Function

\begin{tabular}{|c|c|c|c|c|c|c|c|}
\hline & $c_{1}$ & $a_{1}$ & $c_{2}$ & $a_{2}$ & $c_{3}$ & $a_{3}$ & Residual \\
\hline \hline$\sigma=4$ & 0.49 & 0.75 & 0.49 & 0.75 & 0.03 & 0.16 & $4.6810^{-5}$ \\
\hline$\sigma=6$ & 0.38 & 0.31 & 0.56 & 1.21 & 0.06 & 0.07 & $4.2310^{-6}$ \\
\hline$\sigma=8$ & 0.59 & 1.32 & 0.34 & 0.18 & 0.06 & 0.02 & $1.0410^{-4}$ \\
\hline$\sigma=10$ & 0.29 & 0.09 & 0.65 & 1.17 & 0.05 & 0.01 & $7.5310^{-4}$ \\
\hline$\sigma=12$ & 0.04 & 0 & 0.24 & 0.04 & 0.70 & 0.93 & $3.5210^{-3}$ \\
\hline$\sigma=14$ & 0.20 & 0.01 & 0.03 & 0 & 0.72 & 0.64 & $1.0310^{-2}$ \\
\hline$\sigma=16$ & 0.18 & 0.01 & 0.70 & 0.49 & 0.04 & 0 & $1.6710^{-2}$ \\
\hline
\end{tabular}

different throughput if the transmission rate from the sensors is low. Provided that a higher complexity level can be tolerated, the BAN performance is expected to improve if TDMA-like or FDMA-like MAC protocols are used instead of the considered simple Aloha-like protocol.

\section{APPENDIX}

The modeling of slow-scale fading as a log-normal distribution (that is, a zero-mean Gaussian in $\mathrm{dB}$ scale) raises mathematical difficulties, as shown in (14). The complementary cdf of a zero-mean log-normal random variable is

$\zeta(Z \sigma)=\frac{1}{2}+\frac{1}{2} \operatorname{er}\left(\frac{-10 \log _{10} Z}{\sigma \sqrt{2}}\right)$

where $\operatorname{cr} f-\frac{2}{\sqrt{\pi}} \int_{0}^{x} e^{-t^{2}} \mathbf{d} t$ is the error function. The function $\zeta(z \sigma)$ is shown, in Fig. (6), as a function of $\boldsymbol{Z}$ for $\sigma \in\{4,8,12,16\} \mathrm{dB}$. It can be observed that $\zeta(z \sigma)$ (i) saturates for $z \rightarrow \infty$, regardless of the value of $\rho$, and (ii) has the shape of a decreasing exponential function of $Z$ (for a given value of $\sigma$ ).

Fig. (6). The function $\zeta(z \sigma)$ as a function of $z$, considering various values of $\sigma($ in $\mathrm{dB})$.

The $\xi$ function can be approximated with a linear combination of negative exponential functions, as in [16, 17]:

$\zeta(z ; \sigma)=\sum_{k}^{\infty} c_{k} \exp \left(-a_{k} z\right) \approx \sum_{k}^{n} c_{k} \exp \left(-a_{k} z\right)$

where the coefficients $\left\{c_{k}\right\}_{k=1}^{n}$ and $\left\{a_{k}\right\}_{k=1}^{n}$ depend on $\sigma$ and can be determined in a least square sense by means of $q \geq 2 n$ known points of the $\zeta$ function. The Levenberg-Marquardt algorithm $[18,19]$ can be used to determine the coefficients $\left\{c_{k}\right\}$ and $\left\{a_{k}\right\}$ for different values of $\sigma$ and 10000 points over the interval $z \in[0,1000]$. The corresponding values are reported in Table $\mathbf{2}$ along with the corresponding residual sum of squares.

\section{REFERENCES}

[1] E. Reusens, W. Joseph, G. Vermeeren, and L. Martens, “On-body measurements and characterization of wireless communication channel for arm and torso of human," Proceeding. of the 4th International Workshop on Wearable and Implantable Body Sensor Networks (BSN 2007), vol. 13, pp. 264-269, 2007.

[2] J. M. Choi, H.-J. Kang, and Y.-S. Choi, "A study on the wireless body area network applications and channel models," in Proceedings of the 2008 Second International Conference on Future Generation Communication and Networking. Washington, DC, USA, 2008.

[3] N. Katayama, K. Takizawa, T. Aoyagi, J.-I. Takada, H.-B. Li, and R. Kohno, "Channel model on various frequency bands for wearable body area network," IEICE Transactions on Communications, vol. E92.B, no. 2, pp. 418-424, 2009.

[4] A. Fort, C. Desset, P. De Doncker, P. Wambacq, and L. Van Biesen, "An ultra-wideband body area propagation channel modelfrom statistics to implementation," IEEE Transactions on Microwave Theory and Techniques, vol. 54, no. 4, pp. 1820-1826, 2006.

[5] H. Sawada, J. Takada, S. Choi, K. Yazdandoost, and R. Kohno, "Review of body area network channel model," in Proceeding of IEICE General Conference, 2007.

[6] J. Ryckaert, P. D. Doncker, R. Meys, A. de Le Hoye, and S Donnay, "Channel model for wireless communication around human body," IEEE Electronic Letters, vol. 40, no. 9, pp. 543-544, 2004.

[7] P. Gupta and P. R. Kumar, "The capacity of wireless networks," IEEE Transactions on Information Theory, vol. 46, no. 2, pp. 388404, 2000

[8] X. Liu and M. Haenggi, "Throughput analysis of random and regular networks," EURASIP Journal on Wireless Communications and Networking, vol. 4, pp. 554-564, 2005.

[9] S. van Roy, C. Oestges, F. Horlin, and P. De Doncker, "A comprehensive channel model for uwb multisensor multiantenna body area networks," IEEE Transactions on Antennas and Propagation, vol. 58, no. 1, pp. 163-170, 2010.

[10] S. van Roy, C. Oestges, F. Horlin, and P. De Doncker, "Propagation modeling for uwb body area networks: Power decay and multi-sensor correlations," in Proceeding. of the IEEE 10th International Symposium on Spread Spectrum Techniques and Application, 2008, pp. 649-653.

[11] J. ichi Takada, T. Aoyagi, K. Takizawa, N. Katayama, H. Sawada, T. Kobayashi, K. Y. Yazdandoost, H. bang Li, , and R. Kohno, "Static propagation and channel models in body area," in COST 2100 6th Management Committee Meeting, TD(08)639, 2008.

[12] A. Goldsmith, Wireless Communications. New York, NY, USA: Cambridge University Press, 2005.

[13] D. Bertsekas and R. Gallager, Data Networks, Prentice-Hall, USA, 1991.

[14] X. Liu and M. Haenggi, "Throughput analysis of fading sensor networks with regular and random topologies," EURASIP Journal of Wireless Communications Networking, vol. 2005, no. 4, pp. 554$564,2005$. 
[15] Ahmed, M.S. Alam, "Performance evaluation of important ad hoc network protocols", EURASIP Journal on Wireless Communications and Networking, vol. 2006, no. 2, 2006.

[16] Baron G. de Prony, "Essai expérimental et analytique sur les lois de la dilatabilité des fluides élastique et sur celles de la force expansive de la vapeur de l'eau et de la vapeur de l'alkool, à différentes températures," Journal de l'École Polytechnique, vol. 1 , no. 2, pp. 24-76, 1795.
[17] F. G. Lether, "Elementary approximation for erf(x)," Journal of Quantitative Spectroscopy and Radiative Transfer, vol. 49, no. 5, pp. 573-577, 1993.

[18] K. Levenberg, "A method for the solution of certain non-linear problems in least squares," The Quarterly of Applied Mathematics, vol. 2, pp. 164-168, 1944.

[19] D. Marquardt, "An algorithm for least-squares estimation of nonlinear parameters," SIAM Journal on Applied Mathematics, vol. 11, pp. 431-44, 1963.

(c) Dricot et al.; Licensee Bentham Open.

This is an open access article licensed under the terms of the Creative Commons Attribution Non-Commercial License (http://creativecommons.org/licenses/by-nc/3.0/g) which permits unrestricted, non-commercial use, distribution and reproduction in any medium, provided the work is properly cited. 